\title{
Variable Topological Method for Reliability Assessment of Aerospace Vehicle Separation
}

\author{
Wanmeng Zhou, Haiyang $\mathrm{Li}^{*}$ and Feng Huang \\ National University of Defense Technology, 410073 Chang Sha City, Hu Nan Province, China \\ ${ }^{*}$ Corresponding author
}

\begin{abstract}
Keywords: Separation dynamics, Variable topological structure, Reliability assessment, Separation
\end{abstract} internal force, Monte Carlo simulation.

\begin{abstract}
During the launch of a rocket or missile, certain segments must separate from the main body, and the reliability of this separation can affect the final success of the mission. Most reports on the analysis of the specific separation structures employ specific finite element software programs; moreover, although most studies on reliability focus on the simple dynamics of the segments, few consider the separation dynamics in the distribution reliability analysis. Thus, a generalized variable topological dynamics-based aerospace separation reliability analysis method is proposed in this paper. With the exception of a few basic constraints imposed by external forces, a few internal forces in the separation structure derived from the spring oscillator assumption were also included as variable topological constraints. As the craft separated, the variable topological structure constraints changed according to the transfer conditions. The separation reliability criterion was established after modeling the separation dynamics and used to derive the reliability model and the distribution hypothesis test to estimate the separation reliability. Finally, the Monte Carlo separation simulation was conducted to verify the reliability analysis method. The results indicate that the method can effectively estimate the different phases of the separation process and verify the separation reliability of the aerospace vehicle.
\end{abstract}

\section{Introduction}

The launch of an aerospace vehicle such as rocket and missile involves the separation of segments no longer required for normal operation. The success of the separation is determined by the time to separate, and the motion states experienced during the separation. No separation, or incomplete separation, may result in uncontrollable attitudes and damaged structures, which could cause the mission to fail. Therefore, the separation process must be reliable; reliability can be defined as the capability to perform the desired functions under the demand conditions. In general, the basic requirements for reliable separation are stable fixation before the separation, and subsequent separation without severe vibrations.

Research on the separation system to date has tended to focus on the specific disciplines and domains, including the separation structure design and failure analysis [1], atmospheric static pressure and aerodynamics regulation during the separation [2], multi-rigid-body motion and collision analysis via Monte Carlo simulation [3], the use of finite elements methods to evaluate the static and dynamic properties of a separation structure in an electrodynamic and flexible multi-body system [4], and the control system design of the separation structure [5].

Studies on separation reliability tend to include specific partial or general dynamic simulations. Specific partial analysis studies investigated the reliability with respect to multistage separation [6], nose cap separation [7], submarine missile and carrier separation [8], and trail cover separation [9]. Alternatively, general dynamic simulations are usually conducted using professional simulation software. Gong utilized failure mode and effects analysis of the retro rockets using the algorithm presented by Adams [10]. Zhu used a multi-software platform to analyze the fairing separation process beginning from the unlocking of the fastening device to the rotation of fairing around the hinges [11]. 
The above-mentioned studies indicate that, although there is a large amount of research on the separation structure of aerospace vehicles or the reliability analysis of the separation process, few of these researchers have proposed a general method to concurrently analyze the separation process and separation reliability. The studies on reliability to date have either oversimplified the separation process by modeling the separation structure as an instantaneous force or impulse deviation, or entailed highly complex simulations in which the separation reliability analysis was performed by employing multiple software programs, which proved to be an inflexible and time expensive process. Thus, the aim of this study is to propose a more widely applicable method for separation reliability analysis in which the separation process and reliability are both adequately considered.

\section{Variable Topological Structure Dynamics Modeling}

\section{Basic Topological Constraint}

The two separation segments are assumed as rigid bodies. Different separation interaction forces can be considered as different types of hinge joint constraints, and action points of hinge joint loads are equivalent to the geometrical connection points on the upper and lower separation segments. Because the launch vehicle is not directly connected to any other rigid bodies, an imaginary hinge is implemented in this system. The rigid bodies of the separation system can form a tree system [12], which can be illustrated as is shown in Fig. 1. The launch coordinates are selected as zero rigid body B0. The imaginary hinge $\mathrm{O} 1$ represents the six degrees of freedom connecting $\mathrm{B} 0$ and the separation bodies B1 and B2. The constraining joint between the lower B1 and upper B2 is expressed as O2.

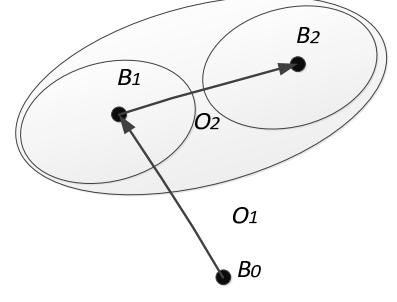

Figure 1. Illustration of the vehicle separation system

It should be noted that the constant constraint in this system is a basic constraint of the entire separation system throughout the separation process, and is expressed as $\boldsymbol{\Phi}_{\boldsymbol{\theta}}(\boldsymbol{x}, t)$, where $\boldsymbol{\Phi}_{\boldsymbol{0}} \in \mathrm{i}^{l^{l_{0}}}$ and $l_{0}$ represent the dimensions of the constraints. Other additional constraints such as fix lock, guide pin, guide block, and rotational hinge, differ from the basic constraints in that they vary according to the changing topological structure. The set of all additional constraints can be expressed as $\Phi_{P}$, i.e., $\boldsymbol{\Phi}_{P}=\left\{\boldsymbol{\Phi}_{1}, \boldsymbol{\Phi}_{2}, \mathrm{~L}, \boldsymbol{\Phi}_{l}, \mathrm{~L}, \boldsymbol{\Phi}_{s}\right\}$, which represent for the topological structure of the system. As an example, when the system enters state $\iota$, the corresponding constraint $\boldsymbol{\Phi}_{\imath}$ in $\boldsymbol{\Phi}_{P}$ is activated, and a new topological structure is established. The imaginary hinge $\mathrm{O} 1$ between the zero-rigid body B0, and the separation vehicle, is considered as the external load. The states of the intact vehicle are given in the launch coordinates system $o_{L} x_{L} y_{L} z_{L}(\mathrm{~L})$. The origin is the launch point B0; the x-axis and y-axis are oriented toward the launch azimuth and vertical direction of the local horizontal plane, respectively, whereas the z-axis is subjected to the right-hand regulation. The separation time is relatively short, and the launch coordinates system is thus assumed to be an inertial coordinate system. The states of the separation motion are expressed in the separation inertial coordinates system (SI). After separation, the motion is expressed in a separation coordinates system $o_{s} x_{S} y_{S} z_{S}$. The origin of this system is the center of the separation plane, and the orientations of all axes are parallel to the upper and lower segments of the body coordinates system at the beginning of separation.

The kinematic and dynamic equations for the lower segment are equivalent to those of the upper segment, as they can be obtained by substituting the subscript $u$ with $d$. Thus, the dynamic and kinematic equations are given by 


$$
\frac{d \boldsymbol{r}_{C u}}{d t}=\boldsymbol{v}_{C u}, m_{u} \frac{d^{2} \boldsymbol{r}_{C u}}{d t^{2}}=\boldsymbol{\Phi}_{F u 0}(\boldsymbol{r}, t)+\boldsymbol{\Phi}_{F u p}(\boldsymbol{r}, t)
$$

where $\boldsymbol{r}_{c u}$ refers to the centroid position in SI coordinates, $\boldsymbol{v}_{c u}$ refers to the velocity in SI coordinates, $m_{u}$ refers to the mass of the vehicle, and $\boldsymbol{\Phi}_{F u 0}(\boldsymbol{r}, t)$ refers to the basic topological constraints, which are given as

$$
\boldsymbol{\Phi}_{F u 0}(\boldsymbol{r}, t)=\boldsymbol{G}_{u}+\boldsymbol{P}_{u r}
$$

where $\boldsymbol{G}_{u}$ is the gravity of the upper segment, and $\boldsymbol{P}_{u r}$ is the thrust of the retro. It should be noted that the thrust constraint from the main motor is applied also on the lower segment.

To prevent attitude singularity of the upper segment, Euler angles and reverse Euler angles are defined in the body coordinates system [13]. The attitude motion equations (i.e., for the first set of Euler angles) are given as

$$
\left(\begin{array}{c}
\dot{\varphi}^{u} \\
\dot{\psi}^{u} \\
\dot{\gamma}^{u}
\end{array}\right)=\left(\begin{array}{c}
\omega_{u y} \sin \gamma^{u}+\omega_{u z} \cos \gamma^{u} \\
\frac{\omega_{u y} \cos \gamma^{u}-\omega_{u z} \sin \gamma^{u}}{\cos \varphi^{u}} \\
\omega_{u x}-\tan \varphi^{u}\left(\omega_{u y} \cos \gamma^{u}-\omega_{u z} \sin \gamma^{u}\right)
\end{array}\right)
$$

where an attitude is defined as a change from the upper-body coordinate system to the SI system following a 2-3-1 rotational sequence (yaw $\psi^{u}$-pitch $\varphi^{u}$-roll $\gamma^{u}$ ), and $\varphi^{u} \in\left[-90^{\circ},+90^{\circ}\right]$, $\psi^{u} \in\left(-180^{\circ},+180^{\circ}\right], \gamma^{u} \in\left(-180^{\circ},+180^{\circ}\right]$.

The attitude dynamics functions are given as

$$
\boldsymbol{I}_{u} \frac{d \boldsymbol{\omega}_{u}}{d t}+\boldsymbol{\omega}_{u} \times\left(\boldsymbol{I}_{u} \cdot \boldsymbol{\omega}_{u}\right)=\boldsymbol{\Phi}_{M u 0}(\boldsymbol{r}, t)+\boldsymbol{\Phi}_{M u p}(\boldsymbol{r}, t)
$$

where $\boldsymbol{I}_{u}$ refers to the upper rotational inertia, and $\boldsymbol{\Phi}_{M u 0}(\boldsymbol{r}, t)$ refers to the basic topological constraints, which are defined as follows:

$$
\boldsymbol{\Phi}_{M u 0}(\boldsymbol{r}, t)=\boldsymbol{M}_{u r}
$$

where $\boldsymbol{M}_{u r}$ is the moment of the retro.

The intact vehicle motion equations are given as

$$
\frac{d \boldsymbol{r}_{L c}}{d t}=\boldsymbol{v}_{L c}, m_{c} \frac{d^{2} \boldsymbol{r}_{L c}}{d t^{2}}=\boldsymbol{T}_{L S} \boldsymbol{\Phi}_{F u}(\boldsymbol{r}, t)+\boldsymbol{T}_{L S} \boldsymbol{\Phi}_{F d \theta}(\boldsymbol{r}, t)
$$

where $m_{c}=m_{u}+m_{d}$, and $\boldsymbol{T}_{L S}$ is the L to SI translation matrix. The attitude dynamics reflected in the intact body coordinate system are identical to those of the upper segment, and are given as

$$
\boldsymbol{I}_{c} \frac{d \boldsymbol{\omega}_{c}}{d t}+\boldsymbol{\omega}_{c} \times\left(\boldsymbol{I}_{c} \cdot \boldsymbol{\omega}_{c}\right)=\boldsymbol{\Phi}_{M u \theta}(\boldsymbol{r}, t)+\boldsymbol{\Phi}_{M d \theta}(\boldsymbol{r}, t)
$$

where the rotational inertia $\boldsymbol{I}_{c}$ of the intact body is given as

$$
\boldsymbol{I}_{c}=\boldsymbol{I}_{u}+\boldsymbol{I}_{d}+m_{u}\left[\begin{array}{ccc}
y_{c u}^{2}+z_{c u}^{2} & -x_{c u} y_{c u} & -x_{c u} z_{c u} \\
-x_{c u} y_{c u} & x_{c u}^{2}+z_{c u}^{2} & -y_{c u} z_{c u} \\
-x_{c u} z_{c u} & -y_{c u} z_{c u} & x_{c u}^{2}+y_{c u}^{2}
\end{array}\right]+m_{d}\left[\begin{array}{ccc}
y_{c d}^{2}+z_{c d}^{2} & -x_{c d} y_{c d} & -x_{c d} z_{c d} \\
-x_{c d} y_{c d} & x_{c d}^{2}+z_{c d}^{2} & -y_{c d} z_{c d} \\
-x_{c d} z_{c d} & -y_{c d} z_{c d} & x_{c d}^{2}+y_{c d}^{2}
\end{array}\right]
$$

\section{Variable Topological Loads Constraint}

The forces and moments for the variable topological structure change according to the transform condition. The variable topological loads constraint $\boldsymbol{\Phi}_{p}(\boldsymbol{r}, t)$ is given as

$$
\boldsymbol{\Phi}_{p}(\boldsymbol{r}, t)=\boldsymbol{F}_{s}+\boldsymbol{F}_{c}+\boldsymbol{R}
$$


where $\boldsymbol{F}_{s}$ is the pulling resistance, $\boldsymbol{F}_{c}$ is the connecting constraint force, and $\boldsymbol{R}$ is the aerodynamic force. The variable moment constraint $\boldsymbol{\Phi}_{M_{p}}(\boldsymbol{r}, t)$ is defined as follows:

$$
\boldsymbol{\Phi}_{M_{p}}(\boldsymbol{r}, t)=\boldsymbol{M}_{\mathrm{s}}+\boldsymbol{M}_{c}+\boldsymbol{M}_{R}
$$

where $\boldsymbol{M}_{s}$ is the pulling moment, $\boldsymbol{M}_{c}$ is the connecting constraint moment, and $\boldsymbol{M}_{R}$ is the aerodynamic moment.

The separation system has $n_{x}$ groups of connecting rods, which generate the pulling resistance. The connecting points $x_{i}\left(i=1, . ., n_{x}\right)$ coincide with the installation site of the pre-separation upper and lower segments. During the separation, the position of $\boldsymbol{x}_{i}$ in SI is expressed as $\boldsymbol{r}_{x i}$. The relative position of the upper and lower connecting points can then be expressed as $\boldsymbol{d}_{u d i}=\boldsymbol{r}_{u x i}-\boldsymbol{r}_{d x i}$. The pulling force on the upper segment is given as

$$
\boldsymbol{F}_{u s}=\sum_{i=1}^{n_{x}} \boldsymbol{F}_{u s i}=\sum_{i=1}^{n_{x}}-f_{s i}\left(\left|\boldsymbol{d}_{\text {udi }}\right|\right) \frac{\boldsymbol{d}_{u d i}}{\left|\boldsymbol{d}_{u d i}\right|}
$$

The force on the lower segment is $\boldsymbol{F}_{d s}=-\boldsymbol{F}_{u s}$. Then, the pulling moment can be expressed as

$$
\boldsymbol{M}_{u s}=\sum_{i=1}^{n_{x}} \boldsymbol{r}_{x i}^{u} \times \boldsymbol{T}_{u S} \boldsymbol{F}_{u s i}, \boldsymbol{M}_{d s}=\sum_{i=1}^{n_{x}} \boldsymbol{r}_{x i}^{d} \times \boldsymbol{T}_{d S} \boldsymbol{F}_{d s i}
$$

where $\boldsymbol{r}_{x i}^{u}$ and $\boldsymbol{r}_{x i}^{d}$ refer to the centroid position, $\boldsymbol{T}_{u s}$ and $\boldsymbol{T}_{d s}$ refer to the transform matrix, from an SI system to the respective body coordinates system. $f_{s i}\left(d_{i}\right)$ is the value of the force, which can be expressed via an interpolation function or step function. If the value is negative, the structure is determined to be the spring push rod, which provides the mechanical separation impulse.

Connecting constraints are generated by connecting devices such as a guide plug, which is illustrated in Fig. 2. Because other connecting devices have similar properties, guide plugs are used as a representative connecting device in this study; the forces and moments on guide plugs are subsequently introduced and discussed. Each group of guide plugs has two feature points $\boldsymbol{x}_{i}(i=1,2)$, which are coincident before the separation. During the separation process, point $\boldsymbol{x}_{2}^{u}$ lies between $\boldsymbol{x}_{1}^{d}$ and $\boldsymbol{x}_{2}^{d}$, and point $\boldsymbol{x}_{1}^{d}$ is located between $\boldsymbol{x}_{1}^{u}$ and $\boldsymbol{x}_{2}^{u}$.
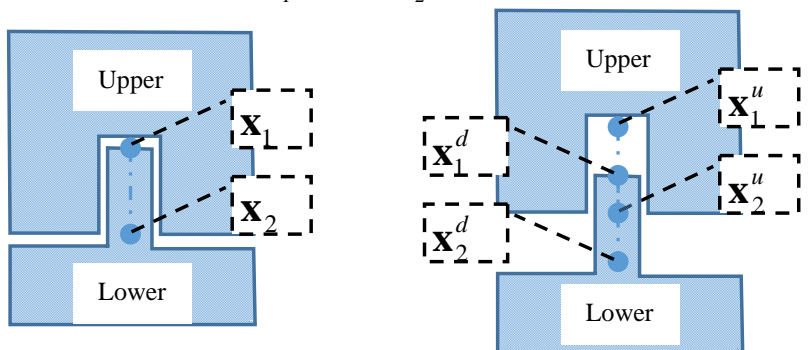

Figure 2. Characteristic points of guide plugs during separation

The constraints incorporated into the equations are replaced by the spring oscillator. The feature points $\boldsymbol{x}_{2}^{u}$ and $\boldsymbol{x}_{1}^{d}$ are used to represent two spring oscillators. $\boldsymbol{r}_{x 1}^{u}$ and $\boldsymbol{r}_{x 2}^{u}$ refer to the upper feature-point positions in the body coordinate system and are expressed as $\boldsymbol{r}_{u \times 1}$ and $\boldsymbol{r}_{u \times 2}$ in SI. Similarly, $\boldsymbol{r}_{x 1}^{d}$ and $\boldsymbol{r}_{x 2}^{d}$ represent the lower feature points in the body coordinate system, and are expressed as $\boldsymbol{r}_{\mathrm{dx1}}$ and $\boldsymbol{r}_{d \times 2}$ in SI. The respective lateral displacements of $\boldsymbol{x}_{1}^{d}$ and $\boldsymbol{x}_{2}^{u}$ can be given as

$$
\begin{aligned}
& \boldsymbol{d}_{1}^{d}=\left(\boldsymbol{r}_{d \times 1}-\boldsymbol{r}_{u \times 1}\right)-\left(\left(\boldsymbol{r}_{d \times 1}-\boldsymbol{r}_{u \times 1}\right) \cdot \boldsymbol{l}_{u}\right) \boldsymbol{l}_{u} \\
& \boldsymbol{d}_{2}^{u}=\left(\boldsymbol{r}_{u \times 2}-\boldsymbol{r}_{d \times 2}\right)-\left(\left(\boldsymbol{r}_{u \times 2}-\boldsymbol{r}_{d \times 2}\right) \cdot \boldsymbol{l}_{d}\right) \boldsymbol{l}_{d}
\end{aligned}
$$


where characteristic length $l_{12}=\left|\boldsymbol{r}_{u \times 1}-\boldsymbol{r}_{u \times 2}\right|=\left|\boldsymbol{r}_{d \times 1}-\boldsymbol{r}_{d \times 2}\right|$,upper characteristic direction $\boldsymbol{l}_{u}=\frac{\boldsymbol{r}_{u \times 1}-\boldsymbol{r}_{u \times 2}}{l_{12}}$, and lower characteristic direction $\boldsymbol{l}_{d}=\frac{\boldsymbol{r}_{d \times 1}-\boldsymbol{r}_{d \times 2}}{l_{12}}$. The axial distances of $\boldsymbol{x}_{2}^{u}$ and $\boldsymbol{x}_{1}^{d}$ are defined as $u_{a}=\left(\boldsymbol{r}_{d \times 1}-\boldsymbol{r}_{u \times 2}\right) \cdot \boldsymbol{l}_{d}$.

The constraint on the upper segment is given as

$$
\boldsymbol{F}_{u c}=\boldsymbol{F}_{u c 1}+\boldsymbol{F}_{u c 2}-F_{s} \boldsymbol{l}_{u}
$$

where $\boldsymbol{F}_{u c 2}=-k_{2} \boldsymbol{d}_{2}^{u}-c_{2} \dot{\boldsymbol{d}}_{2}^{u}$, and $\boldsymbol{F}_{u c 1}=k_{1} \boldsymbol{d}_{1}^{d}+c_{1} \dot{\boldsymbol{d}}_{1}^{d}$.The force of friction is $F_{s}=f_{s}\left(\left|\boldsymbol{F}_{u c 1}\right|+\left|\boldsymbol{F}_{u c 2}\right|\right)$, where $f_{s}$ is the friction coefficient, $k$ is the stiffness coefficient, and $c$ is the damping coefficient. For the characteristic points, the default direction of motion is one that involves the points moving away from each other. The constraint on the lower segment can be derived as $\boldsymbol{F}_{\mathrm{dc}}=-\boldsymbol{F}_{u c}$.

Considering the relative movement and the force of the guide plug, the constraint moments of two segments are derived as

$$
\begin{aligned}
& \boldsymbol{M}_{u c}=\boldsymbol{r}_{x 2}^{u} \times \boldsymbol{T}_{u S} \boldsymbol{F}_{u c 2}+\left(\frac{u_{a}}{l_{12}} \boldsymbol{r}_{x 1}^{u}+\frac{l_{12}-u_{a}}{l_{12}} \boldsymbol{r}_{x 2}^{u}\right) \times \boldsymbol{T}_{u s} \boldsymbol{F}_{u c 1}-\boldsymbol{r}_{x 2}^{u} \times F_{s} \boldsymbol{T}_{u S} \boldsymbol{I}_{u} \\
& \boldsymbol{M}_{d c}=\boldsymbol{r}_{x 1}^{d} \times \boldsymbol{T}_{d S} \boldsymbol{F}_{\mathrm{dc1}}+\left(\frac{l_{12}-u_{a}}{l_{12}} \boldsymbol{r}_{x 1}^{d}+\frac{u_{a}}{l_{12}} \boldsymbol{r}_{x 2}^{d}\right) \times \boldsymbol{T}_{d S} \boldsymbol{F}_{d c 2}+\boldsymbol{r}_{x 2}^{d} \times F_{s} \boldsymbol{T}_{d s} \boldsymbol{l}_{d}
\end{aligned}
$$

After totally separation (i.e. $u_{a} \leq 0$ ), the value of the constraints can then be set to zero.

\section{Separation Reliability Simulation}

\section{Reliability Assessment Experiment Design}

The probable deviation factors include installation deviations, errors in the initial positions and angles, the deviation of the standard thruster, aerodynamics errors, wind field influence, and other unknown disturbances. All these parameter deviations and process disturbances can result in deviation from the desired path of motion and generate uncertainty.

Generalized continuous random variables may satisfy the conditions for various distributions. However, for the separation system, only commonly applicable distributions such as normal and uniform are considered. The deviation factors are chosen on the basis of the results of a previously published report [6]. In order to detect any collisions, $n_{u}$ and $n_{d}$ points are set on the separation segments, and $N$ groups of these points are selected as the judgment points.

The centroid positions $\boldsymbol{r}_{c u}$ and $\boldsymbol{r}_{c d}$ of the upper and lower segments can be obtained via the variable topological separation dynamics at any time throughout the process. $N$ points are randomly selected from both the envelopes, which are subsequently formed into $N$ groups of feature points $\boldsymbol{r}_{u i}\left(x_{u i}, y_{u i}, z_{u i}\right)$ and $\boldsymbol{r}_{d i}=\left(x_{d i}, y_{d i}, z_{d i}\right)$; the distance between the feature points is expressed as $d_{u d}=\left\|\boldsymbol{r}_{u i}-\boldsymbol{r}_{d j}\right\|_{2}$. Then, the following quantities are considered:

(1) The minimum of all groups of distances at the reference time $t_{3}$ in the $k_{t h}$ experiment is expressed as $d_{i}^{k}=\min _{1 \leq i \leq N}\left(\left\|r_{u i}^{k}\left(t_{3}\right)-\boldsymbol{r}_{d i}^{k}\left(t_{3}\right)\right\|\right)$;

(2) The minimum of all groups of distances during the separation process in the $k_{t h}$ experiment is expressed as $d_{i \min }^{k}=\min _{1 \leq i \leq N}\left(\left\|\boldsymbol{r}_{u i}^{k}-\boldsymbol{r}_{d i}^{k}\right\|\right)$.

where $k=1, \ldots, N_{T}$. Based on the statistics of the abovementioned characteristic distances obtained from $N_{T}$ simulations, the reliability assessment can be calculated by assessing the $d_{i}^{k}>$ ò (or $d_{i \min }^{k}>$ ò ), where ò is the safe distance. 
The distribution of the characteristic distance is assumed as $d_{i} \sim N\left(\mu, \sigma^{2}\right)$, which can be expressed as $\mathrm{H}_{0}: d_{i} \sim N\left(\mu, \sigma^{2}\right)$. The statistical analysis procedure is explained below.

Step1: Divide the value range $a_{y}$ of characteristic distance $d_{i}$ into $N_{y}$ mutually disjoint sections $A_{j}=\left(a_{y}^{j}, a_{y}^{j+1}\right], j=2, \cdots, N_{\mathrm{y}}$. When $j=1, A_{1}=\left[a_{y}^{1}, a_{y}^{2}\right]$. The number of the specific case that the characteristic distance $d_{i}$ lies in the section $A_{j}$, and is expressed as $n_{j}$;

Step2: Under the hypothesis of $\mathrm{H}_{0}$, the maximum likelihood estimation (MLE) of $\mu$ and $\sigma^{2}$ are determined as follows:

$$
\hat{\mu}=\frac{1}{N_{T}} \sum_{k=1}^{N_{T}} d_{i}^{k}=\overline{d_{i}}, \quad \hat{\sigma}^{2}=\frac{1}{N_{T}} \sum_{i=1}^{N_{T}}\left(d_{i}^{k}-\overline{d_{i}}\right)^{2}
$$

Step3: Calculate the frequency estimation of each section $A_{j}$ by solving

$$
\hat{p}_{j}=\Phi\left(\frac{a_{y}^{j+1}-\hat{\mu}}{\hat{\sigma}}\right)-\Phi\left(\frac{a_{y}^{j}-\hat{\mu}}{\hat{\sigma}}\right)
$$

where $\Phi(x)=\frac{1}{\sqrt{2 \pi}} \int_{-\infty}^{x} e^{-\frac{t^{2}}{2}} d t$.

Step4: Calculate the Pearson statistics,

$$
\chi^{2}=\sum_{j=1}^{r} \frac{\left(n_{j}-N_{T} \hat{p}_{j}\right)^{2}}{N_{T} \hat{p}_{j}}
$$

Step5: Calculate the chi-square probability,

$$
F\left(N_{y}-k-1, \chi^{2}\right)=\frac{\int_{0}^{\frac{\chi^{2}}{2}} t^{\frac{N_{y}-k-1}{2}-1} e^{-t} d t}{\int_{0}^{\infty} t^{\frac{N_{y}-k-1}{2}-1} e^{-t} d t}
$$

where the number of estimated parameters $\mathrm{k}=2$.

Step6: Calculate the significance level and reliability assessment as follows:

$$
\begin{aligned}
& \alpha_{1}=1-F\left(N_{y}-k-1, \chi^{2}\right) \\
& \hat{R}=\int_{\dot{0}}^{a_{y}^{N_{y}}} \frac{1}{\sqrt{2 \pi} \hat{\sigma}} e^{-\frac{\left(d_{i}-\hat{\mu}\right)^{2}}{2 \hat{\sigma}^{2}}} \mathrm{~d} d_{i}
\end{aligned}
$$

\section{Simulation Results Analysis}

The rocket separation is introduced here to study the separation by using the guide plug as the connecting device. The process is partitioned into three topological states. Initially, the rocket operates in the intact body mode. Under the influence of the lower thruster, retro rockets, and connecting structures, the rocket begins to separate into the upper and lower segments. Both segments finally run without the connecting constraints for some time. The initial simulation time is set as $0 \mathrm{~s}$, and unlock commences at $0.4 \mathrm{~s}$. The simulation step before the occurrence of separation is set as $0.1 \mathrm{~s}$; from 0.4 to $0.5 \mathrm{~s}$, the simulation step is set to $0.001 \mathrm{~s}$ and after $0.5 \mathrm{~s}$, the step is $0.01 \mathrm{~s}$. The remaining force of the main thruster, aerodynamic parameters, and the thrust from two retro rockets are interpolated from data. The connecting devices include four separation rods and three guide plugs. The basic topological structure parameters are listed in Table 1, and the reliability analysis setting parameters are listed in Table 2. The remaining relevant parameters can be found in the Appendix. 
Table 1. Basic topological structure parameters

\begin{tabular}{|c|c|c|}
\hline Characteristics parameters & Design value & Unit \\
\hline Longitude of launch point & 0 & $\circ$ \\
\hline Latitude of launch point & 0 & $\circ$ \\
\hline Azimuth of launch point & 0 & $\circ$ \\
\hline Upper feature area & 0.75 & $\mathrm{~m}^{2}$ \\
\hline Upper feature length & 2.6 & $\mathrm{~m}$ \\
\hline Lower feature area & 0.75 & $\mathrm{~m}^{2}$ \\
\hline Lower feature length & 3.87 & $\mathrm{~m}$ \\
\hline Intact feature area & 0.75 & $\mathrm{~m}^{2}$ \\
\hline Intact feature length & 6.47 & $\mathrm{~m}$ \\
\hline Main motor working time & 0.2 & $\mathrm{~S}$ \\
\hline Main motor operating position & $(-2.01,0,0)$ & $\mathrm{m}$ \\
\hline Main motor tilt angles & $(0,0)$ & $\circ$ \\
\hline Retro rocket ignition time & 0.015 & $\mathrm{~S}$ \\
\hline Ignition delay & 0.006 & $\mathrm{~S}$ \\
\hline Position of retro rocket 1 & $(-0.182,-0.105,0.599)$ & $\mathrm{m}$ \\
\hline Tilt angles of retro rocket 1 & $(180,0)$ & $\circ$ \\
\hline Position of retro rocket 2 & $(-0.182,0.105,0.599)$ & $\mathrm{m}$ \\
\hline Tilt angles of retro rocket 2 & $(180,0)$ & $\circ$ \\
\hline
\end{tabular}

Table 2. Reliability analysis setting parameters

\begin{tabular}{|c|c|c|}
\hline Characteristics parameters & Design value & Unit \\
\hline Feature point 1A & $(0.5,1,1)$ & $\mathrm{m}$ \\
\hline Feature point 1B & $(-0.5,1,1)$ & $\mathrm{m}$ \\
\hline Feature point 2A & $(0.5,1,-1)$ & $\mathrm{m}$ \\
\hline Feature point 2B & $(-0.5,1,-1)$ & $\mathrm{m}$ \\
\hline Feature point 3A & $(0.5,-1,1)$ & $\mathrm{m}$ \\
\hline Feature point 3B & $(-0.5,-1,1)$ & $\mathrm{m}$ \\
\hline Feature point 4A & $(0.5,-1,-1)$ & $\mathrm{m}$ \\
\hline Feature point 4B & $(-0.5,-1,-1)$ & $\mathrm{m}$ \\
\hline Reference time & 0.7 & $\mathrm{~s}$ \\
\hline Safe distance & 0.97 & $\mathrm{~m}$ \\
\hline
\end{tabular}

The number of times that the simulation is run is initially set to 20 . The motion states of the upper segment are shown in Fig. 3.

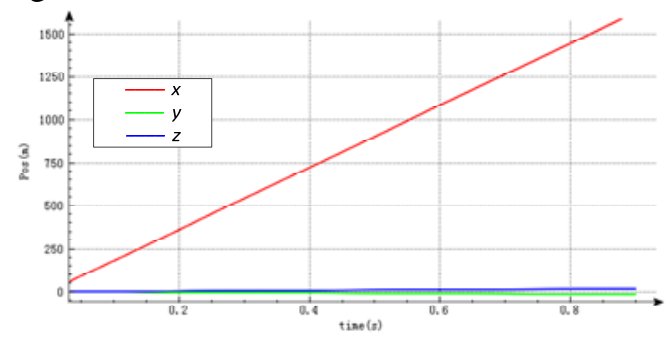

A. Centroid positions of the upper segment in SI

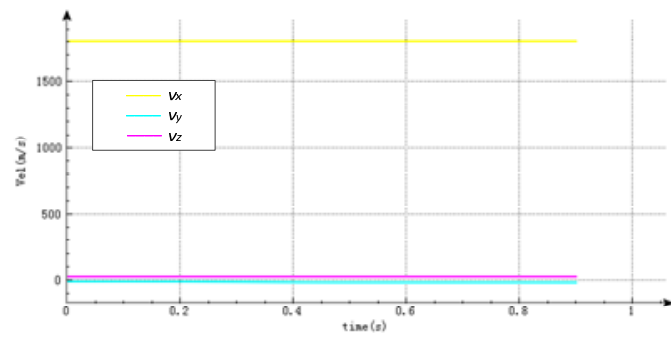

B. Velocities of the upper segment in SI

Figure 3. Historical translational states of the upper segment during separation

Because the main motor installed on the lower segment is mainly oriented along the $\mathrm{x}$-axis in the body coordinate system, the position and velocity along this axis are larger than those along the remaining two axes. Thus, the influence of the deviation in the latter directions is negligible. The rocket is assumed to have a static and stable configuration. Thus, stability can be maintained without the need for external interference under the condition of weak disturbances. The results of the lower segment are similar to those of the upper segment, as shown in Fig. 4. 


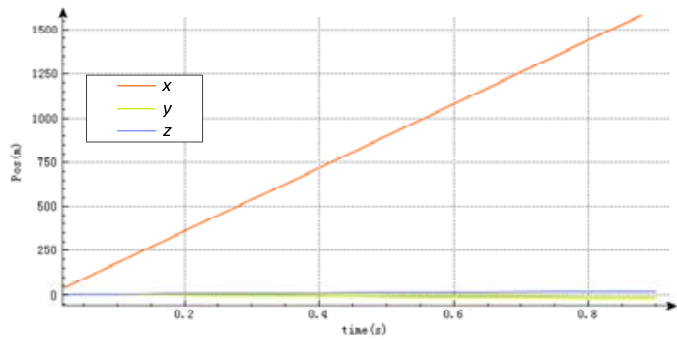

A. Centroid positions of the lower segment in SI

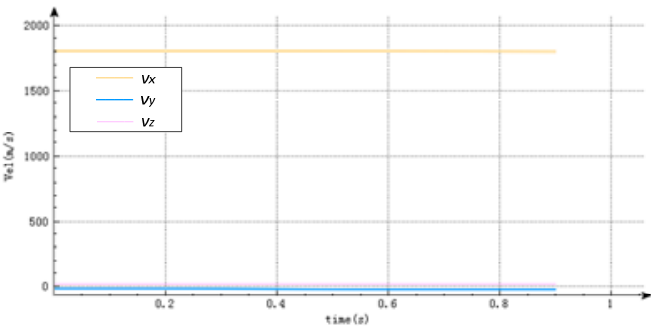

B. Velocities of the lower segment in SI

Figure 4. Historical translational states of the lower segment during separation

The results illustrated in the above figures indicate that, although all states of the lower segment occur in coincidence with those of the upper segment before the separation, the lower segment seems to be more stable than the upper segment. The relationships between pressure center, centroid, and feature length also confirm this disparity in stability.

During the separation process, the distances of four groups of characteristic points are shown in Fig. 5. The distance between the centroids of separation segments is shown in Fig. 6.

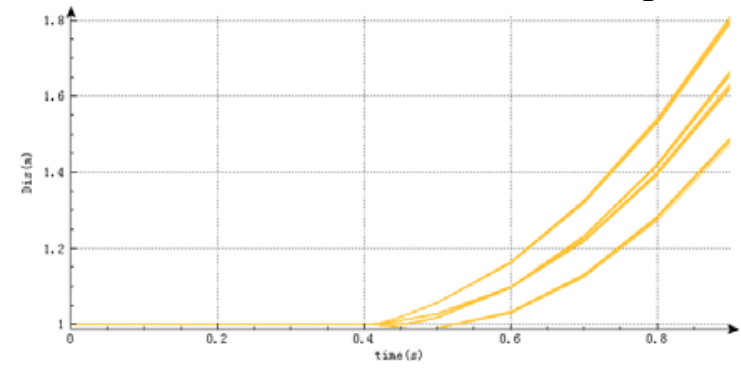

Figure 5. Four groups of characteristic distances in the process of separation

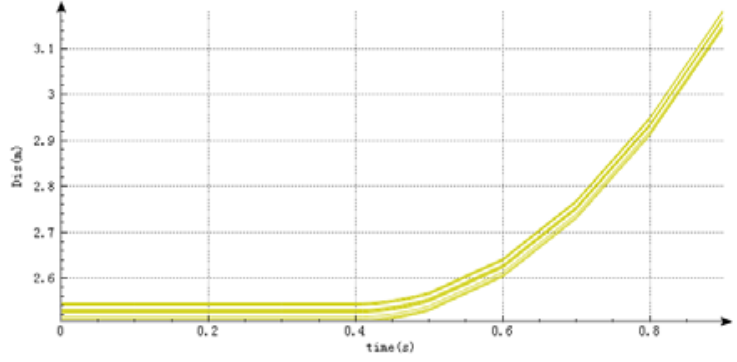

Figure 6. Centroids of the upper and lower distances throughout the process of separation

As was mentioned, the unlock phase begins at $0.4 \mathrm{~s}$. The simulation results of characteristic distances and centroid distances indicate that the upper and lower segments successfully separate. The retro rockets are installed on the lower segment, thereby yielding less influence on the translational motion of the upper segment.

The goodness of fit statistic should have large samples, as the size should exceed 50 for optimal results. The simulation running time is set to 1000; the results are shown in Fig. 7.

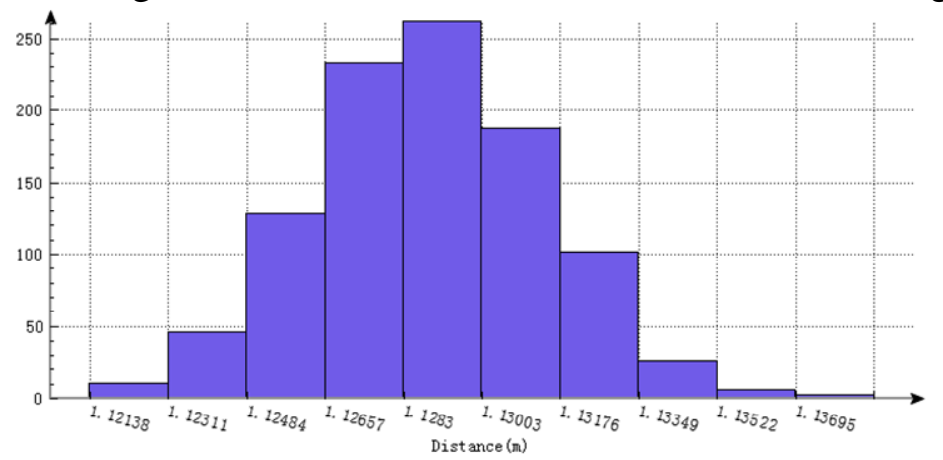

Figure 7. Distribution of the characteristic distance at reference time t3

The results presented in Fig. 7 illustrate the probable normal distribution of distances. The hypothesis is $\mathrm{H}_{0}: r_{r} \sim N\left(\mu, \sigma^{2}\right)$, and the MLE results are as follows: $\hat{\mu}=1.128897$ and $\hat{\sigma}^{2}=6.413809 \mathrm{e}-6$. Under this hypothesis, the Pearson statistics results are determined for $\chi^{2}=4.331$ with a significance level set to 0.05 . The span is divided into ten sections, and the number of estimated parameters is two. The Pearson statistics results for a significance level of 0.05 are as follows:

$\chi_{1-\alpha}^{2}(r-k-1)=\chi_{0.95}^{2}(10-2-1)=\chi_{0.95}^{2}(7)=14.0671$. Thus, the hypothesis is acceptable. 


\section{Conclusions}

The variable topological separation dynamics of aerospace vehicles were discussed in this paper. The process of separation was partitioned into two or three phases according to the topological structure. The variable topological constraints were established as based on the spring oscillator model, and the reliability analysis method was derived from the proposed criterion. The Monte Carlo experiment was conducted in consideration of load deviation, mass, rotational inertia, and initial motion states. The simulation results yielded high separation reliability, thereby demonstrating the efficacy of the proposed method. It should be noted that a comprehensive evaluation of the impact of each of the various deviation factors was not included in the scope of this study. Thus, future research should focus on improving the deviation factor sensitivity to provide more helpful guides for vehicle design.

\section{Acknowledgement}

The authors wish to thank the reviewers of the editorial board, who provided invaluable advice for this paper, in addition to the colleagues and friends of the authors affiliated with the National University of Defense Technology.

\section{Appendix}

Table 3. Topological parameters list

\begin{tabular}{|c|c|c|c|c|c|}
\hline Parameters & Value & Error Type & Factor I & Factor II & Unit \\
\hline Upper mass & 280 & Normal & 0.02 & 0.1 & $\mathrm{~kg}$ \\
\hline Upper centroid position & $0.791,-0.001,0.001$ & Normal & 0.002 & 0.01 & $\mathrm{~m}$ \\
\hline Upper rotational inertia & $6.3,53.5,22.1$ & Normal & 0.002 & 0.01 & $\mathrm{~kg} \cdot \mathrm{m}^{2}$ \\
\hline Upper tensor & $0,0,0$ & Normal & 0.002 & $0.01,0.002,0.01$ & $\mathrm{~kg} \cdot \mathrm{m}^{2}$ \\
\hline Lower mass & 876 & Normal & 30 & 1 & $\mathrm{~kg}$ \\
\hline Lower centroid position & $-1.685,0.028,-0.02$ & Normal & $-0.05,0.01,-0.01$ & 0.01 & $\mathrm{~m}$ \\
\hline Lower rotational inertia & $\begin{array}{c}381.59,745.62, \\
745.62\end{array}$ & Normal & -0.03 & $\begin{array}{c}0.01,-0.03 \\
-0.03\end{array}$ & $\mathrm{~kg} \cdot \mathrm{m}^{2}$ \\
\hline Lower tensor & 0 & Normal & 0.002 & $0.01,0.002,0.01$ & $\mathrm{~kg} \cdot \mathrm{m}^{2}$ \\
\hline $\begin{array}{c}\text { Pitch/yaw/roller initial } \\
\text { attitudes }\end{array}$ & $0.5,0.5,1.5$ & Normal & 0.02 & 0.01 & $\circ$ \\
\hline $\begin{array}{c}\text { Pitch/yaw/roller initial } \\
\text { angular velocities }\end{array}$ & $1.5,0.5,0.5$ & Normal & 0.02 & 0.01 & $\% / \mathrm{s}$ \\
\hline initial positions & $0,8000,0$ & Normal & 20 & 10 & $\mathrm{~m}$ \\
\hline initial velocities & $1800,0,0$ & Normal & 2 & 1 & $\mathrm{~m} / \mathrm{s}$ \\
\hline Retro rocket $1 \sim 2$ thruster & 1 & Extreme & -181 & / & $\mathrm{N}$ \\
\hline Retro rocket 1 2 Impulse & 699 & Extreme & 35 & 1 & $\mathrm{~N} \cdot \mathrm{s}$ \\
\hline Separation connector force & 90 & Normal & 0.02 & 0.1 & $\mathrm{~N}$ \\
\hline \multicolumn{2}{|l|}{ Parameters } & \multicolumn{2}{|l|}{ Value } & \multicolumn{2}{|l|}{ Unit } \\
\hline \multicolumn{2}{|l|}{ Pulling distance } & \multicolumn{2}{|c|}{$0.005 \sim 0.02$} & \multicolumn{2}{|l|}{$\mathrm{m}$} \\
\hline \multicolumn{2}{|c|}{ Separation rod 1 action point } & \multicolumn{2}{|c|}{$2.43 e-6,-0.15,0.08$} & \multicolumn{2}{|l|}{$\mathrm{m}$} \\
\hline \multicolumn{2}{|c|}{ Separation rod 2 action point } & \multicolumn{2}{|c|}{ 2.43e-6,0.15,0.08 } & \multicolumn{2}{|l|}{$\mathrm{m}$} \\
\hline \multicolumn{2}{|c|}{ Separation rod 3 action point } & \multicolumn{2}{|c|}{$2.43 e-6,0.15,-0.08$} & \multicolumn{2}{|l|}{$\mathrm{m}$} \\
\hline \multicolumn{2}{|c|}{ Separation rod 4 action point } & $2.43 e-6,-0$. &,-0.08 & $\mathrm{~m}$ & \\
\hline Guide plug 1 upper pc & & $2.43 e-6,-0$. & $5,0.08$ & $\mathrm{~m}$ & \\
\hline Guide plug 1 lower pc & & $0.01,0.01$ & 0.01 & $\mathrm{~m}$ & \\
\hline Guide plug 1 coefficie & & $0.01,0.01$ & .001 & $\mathrm{~N} / \mathrm{m}, \mathrm{N} /(\mathrm{m} / \mathrm{s}$ & \\
\hline Guide plug 2 upper pc & & $2.43 e-6,0$. &, 0.08 & $\mathrm{~m}$ & \\
\hline Guide plug 2 lower pc & & $0.01,0.01$ & 0.01 & $\mathrm{~m}$ & \\
\hline Guide plug 2 coefficie & & $0.01,0.01$ & .001 & $\mathrm{~N} / \mathrm{m}, \mathrm{N} /(\mathrm{m} / \mathrm{s}$ & \\
\hline Guide plug 3 upper pc & & $2.43 e-6,0.1$ &,-0.08 & $\mathrm{~m}$ & \\
\hline Guide plug 3 lower po & & $0.01,0.01$ & 0.01 & $\mathrm{~m}$ & \\
\hline Guide plug 3 coefficie & & $0.01,0.01$ & .001 & $\mathrm{~N} / \mathrm{m}, \mathrm{N} /(\mathrm{m} / \mathrm{s}$ & \\
\hline
\end{tabular}




\section{References}

[1] Q. Lin, H. Nie, J. Ren, J. B. Chen, Investigation on design and reliability analysis of a new deployable and lockable mechanism, Acta Astronautica, 73 (2012) 183-192.

[2] W. C. Woods, S. D. Holland, M. DiFulvio, Hyper-X stage separation wind-tunnel test program, Journal of Spacecraft and Rockets, 38 (2001) 811-819.

[3] J. Roshanian, M. Talebi, Monte Carlo simulation of stage separation dynamics of a multistage launch vehicle, Applied Mathematics and Mechanics, 29 (2008) 1411-1426.

[4] Q. Cui, Dynamical simulation of separation and reliability analysis for aircraft fairing, Dissertation in Harbin Institute of Technology, 2011.

[5] S. Lui, S. J. Xu, Attitude controller design for combined spacecraft after separation, Aerospace Control and Application, 35 (2009) 35-45.

[6] J. Singaravelu, D. Jeyakumar, B. N. Rao, Taguchi's approach for reliability and safety assessments in the stage separation process of a multistage launch vehicle, Reliability Engineering and System Safety, 94 (2009) 1526-1541.

[7] Y. J. Zhang, Q. Sun, Reliability analysis on separtion trajectory of integral nose cap, Journal of Projectiles, Rockets, Missiles and Guidance, 31 (2011) 19-22.

[8] G. Hui, Y. W. Zhang. Modeling and simulation of the probability of submarine-lauched missile carrier smashing the submarine, Journal of Northwestern Polytechnical University, 23 (2005) 23-27.

[9] R. Y. Jia, T. Wang, Z. Y. Jiang, F. Hu, W. H. Zhang, Uncertainty analysis of the rocket trail cover separation, Journal of National Unvierstiy of Defense Technology, 36 (2014) 88-92.

[10]Q. Gong, J. G. Zhang, F. Chen, W. Z. Li, X. Liu, Separation reliability analysis and failure simulation for a launch vehicle, Spacecraft Engineering, 18 (2009) 60-63.

[11]X. C. Zhu, H. Y. Li, T. X. Yu, B. F. Song, Research on reliability analysis for low-altitude and high-speed payload fairing separation, International Conference on Quality, Reliability, Risk, Maintenance and Safety Engineering IEEE, (2013) 90-94.

[12]R. E. Roberson, R. Schwertassek, Dynamics of Multibody Systems. Berlin: Springer-Verlag, 2012.

[13] W. Zhou, X. J. Zhang, B. H. Kou, Z. Z. Qi. The application of the dual-euler method for overcoming the singularity of Euler equation in parachute-missile system, Spacecraft recovery and Remote Sensing, 24 (2003) 4-8. 